\title{
Micromechanical modelling of cemented sands under low amplitude oscillations
}

\author{
C. S. CHANG,* A. MISRA* and S. S. SUNDARAM*
}

Microstructural continuum modelling is applied to study the mechanical properties of cemented sands under low amplitude oscillatory loading. The effect of cement in sand is accounted for by considering adhesion in addition to friction at the inter-particle contact. Under the assumption that cemented sands are statistically isotropic random packings of equal spheres, a closed form relationship is derived for the initial shear modulus of cemented sands under isotropic confining stress. Also, the secant moduli and damping ratios are computed numerically for various strain amplitudes. The influence of degree of cementation, initial confining stress, particle size, void ratio and strain amplitude on the mechanical properties of cemented sands are investigated. Comparisons of the theoretically predicted results are made with experimental measurements.

KEYWORDS: constitutive relations; fabric/structure of soils; granular materials; microseopy; shear modulus; stiffness.
Un modele à continuum microstructural est utilisé pour étudier les propriétés mécaniques des sables cimentés sous chargement oscillatoire de base amplitude. L'effet de la cimentation du sable est prise en compte en considérant l'adhésion en plus du frottement au contact interparticulaire. Avec l'hypothèse que les sables cimentés représentent des tassements fortuits statistiquement isotropes des sphères égales, une forme fermée de relation est calculée pour le module initial de cisaillement des sables cimentés sous contrainte de confinement isotrope. Les modules de sécante et les rapports d'amortissement sont calculés de façon numérique pour de différentes amplitudes de déformation. L'article examine aussi l'influence exercée par le degré de cimentation, la contrainte initiale de confinement, la grandeur des particules, l'indice des vides et l'amplitude de déformation sur les propriétés mécaniques des sables cimentés. Les résultats obtenues sur la base de la théorie sont comparés avec des mesures expérimentales.

\section{INTRODUCTION}

Cemented sands, both natural and artificial, are often encountered in geotechnical engineering. Naturally occurring cemented sands are formed due to the deposition of small amounts of cementing agents like silica, hydrous silicates, hydrous ion oxides, calcite cement, and carbonates deposited at contacts between sand particles. Artificially made cemented sands are produced mostly from admixture soil stabilization. The admixtures used for the purpose of soil stabilization include portland cement, lime, lime-fly ash and bituminous materials.

The role of cement in sand is to create an adhesive bond at the inter-particle contacts. This adhesive bond significantly influences the mechanical properties of cemented sands. In the past few years, research efforts have been devoted to experimental investigations of the mechanical properties of cemented sands under monotonic as well as cyclic loading conditions (Clough, Sitar, Bachus \& Rad, 1981; Acar \& El-Tahir, 1986;

Discussion on this Paper closes 1 October 1990; for further details see $\mathrm{p}$. ii.

* Department of Civil Engineering, University of Massachusetts, Amherst, MA.
Chang \& Woods, 1987; Saxena \& Lastrico, 1978; Dupas \& Pecker, 1979). A number of experimental results are available in the literature on the initial moduli, the secant moduli and the damping ratio of cemented sands from tests conducted in resonant column device. However, very few mathematical models can be found in the literature which model these properties for cemented sands. There is a gap between the accumulated knowledge from experimental studies and the ability to understand these phenomena through mathematical modelling.

Owing to the particulate nature, sands carry the applied load through resistance at interparticle contacts. To account for this loadcarrying mechanism in the stress-strain modelling of cemented sands, it is necessary to consider the contact behaviour between particles. Analytical models that consider frictional contact behaviour for random packing of spheres (Chang, 1987), based on the microstructural continuum approach have been found to give reasonable agreement with the experimental measurements for shear modulus of uncemented sands under small strain conditions (Chang \& Misra, 1989a, Chang, Sundaram \& Misra, 1989). For cemented sands the presence of bonding material creates an 
additional adhesive resistance at the interparticle contacts. In this Paper, the microstructural model (Chang, 1987) is extended for cemented sands by considering adhesive resistance in addition to the frictional resistance at the contacts. Based on this. extended analytical model the initial shear modulus, secant shear modulus, and damping ratio for cemented sands are computed for low amplitude oscillatory loading. The results are compared with those measured in a resonant column device. Effects of degree of cementation, initial confining stress, strain amplitude, particle size and void ratio on the properties of cemented sands are discussed.

\section{STRESS-STRAIN RELATIONSHIP FOR PARTICULATES}

Following the microstructural continuum approach for stress-strain modelling, the general relationship between incremental stress $\Delta \sigma_{i j}$ and the incremental strain $\Delta \varepsilon_{\mathbf{k} 1}$ for a particulate medium made of spherical particles can be written as

$$
\Delta \sigma_{\mathrm{ij}}=C_{\mathrm{ijkl}} \Delta \varepsilon_{\mathrm{kl}}
$$

where the fourth rank stiffness tensor $C_{\mathrm{ijkl}}$ of the packing is expressed as a volume average of the contact stiffness considering the packing structure given by (Chang, 1987; Chang \& Misra, 1989b)

$$
C_{\mathrm{ijk} \mathbf{l}}=\frac{1}{2 V} \sum_{\mathrm{n}} \sum_{\mathrm{m}} l_{\mathbf{j}}^{\mathrm{nm}} l_{1}^{\mathrm{nm}} K_{\mathrm{ik}}^{\mathrm{nm}}
$$

In Equation (2), the local contact stiffness $K_{\mathrm{ik}}{ }^{\mathrm{nm}}$, weighted by a local fabric tensor $l_{j}^{\mathrm{nm}} l_{1}^{\mathrm{nm}}$, is summed up over all the contact to represent the stress-strain tensor for the packing. Here, $V$ is a given volume of the material, $K_{\mathbf{i k}}{ }^{\mathrm{nm}}$ is the stiffness of the $m$ th contact of the $n$th particle, and $l_{\mathrm{j}}^{\mathrm{nm}}$ is the branch vector joining the centroid of the $n$th particle to the centroid of its neighbour in contact at the $m$ th contact. The first summation is carried over all the particles in the volume and the

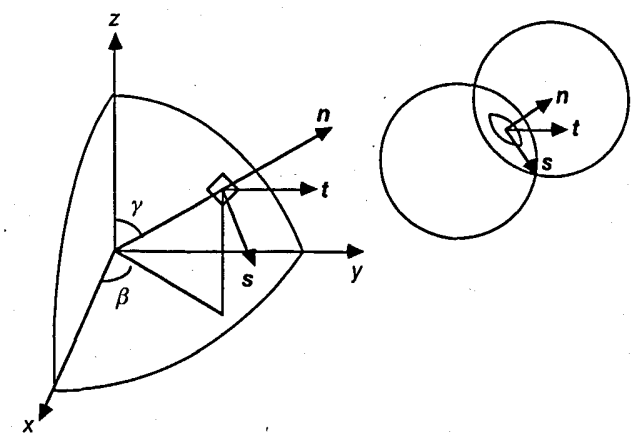

Fig. 1. Local co-ordinate system second over all the contacts for each particle. The usual summation convention for cartesian tensors is assumed for the subscripts. The factor 2 appears in Equation (2) because each contact is counted twice in the double summation process. Equation (2) is arrived at by considering the relationship between the contact forces and stress in the particulate, and the relationship between the relative movement between particles and the strain.

The contact stiffness tensor $K_{\mathrm{ij}}{ }^{\mathrm{nm}}$, in Equation (2), defines the force-displacement relationship at the contact between two particles. Two deformable particles in contact are conceptually treated as two rigid bodies connected by deformable springs (Mindlin \& Deresiewicz, 1953). The relative movement between particles due to the particle deformation at the inter-particle contact is accounted by the spring deformation. The increment of spring deformation, $\Delta \delta_{j} \mathrm{~nm}$, due to an increment of force at the contact, $\Delta f_{i}^{n m}$, is related through the contact stiffness tensor $K_{\mathrm{ij}}{ }^{\mathrm{nm}}$ as

$$
\Delta f_{\mathrm{i}}^{\mathrm{nm}}=K_{\mathrm{ij}}^{\mathrm{nm}} \Delta \delta_{\mathrm{j}}^{\mathrm{nm}}(i, j=x, y, z)
$$

Assuming the shear forces to be independent of the normal deformation and vice versa, the contact stiffness tensor $K_{\mathrm{ij}}^{\mathrm{nm}}$ can be simplified to

$$
K_{\mathrm{ij}}^{\mathrm{nm}}=K_{\mathrm{n}} n_{\mathrm{i}}^{\mathrm{nm}} n_{\mathrm{j}}^{\mathrm{nm}}+K_{\mathrm{r}}\left(s_{\mathrm{i}}^{\mathrm{nm}} s_{\mathrm{j}}^{\mathrm{nm}}+t_{\mathrm{i}}^{\mathrm{nm}} t_{\mathrm{j}}{ }^{\mathrm{nm}}\right)
$$

where $K_{\mathrm{n}}$ and $K_{\mathrm{r}}$ are the normal and the tangential contact stiffnesses respectively. The unit vector $n$ is normal to the contact surface and $n s t$ forms a local co-ordinate system as shown in Fig. 1. Expressed in terms of $\gamma$ and $\beta, n, s$ and $t$ are given by

$$
\begin{aligned}
n & =\sin \gamma \cos \beta i+\sin \gamma \sin \beta j+\cos \gamma k \\
s & =\cos \gamma \cos \beta i+\cos \gamma \sin \beta j-\sin \gamma k \\
t & =-\sin \beta i+\cos \beta j
\end{aligned}
$$

The stress in a particulate medium can be defined as a volume-average of the contact forces, $\Delta f_{\mathrm{j}}^{\mathrm{nm}}$, as (Christoffersen, Mehrabadi \& NematNasser, 1981)

$$
\Delta \sigma_{\mathrm{ij}}=\frac{1}{2 V} \sum_{\mathrm{n}} \sum_{\mathrm{m}} l_{\mathrm{i}}^{\mathrm{nm}} \Delta f_{\mathrm{j}}^{\mathrm{nm}}
$$

where the superscripts refer to the $m$ th contact of the $n$th particle, $l_{\mathrm{i}}^{\mathrm{nm}}$ is the branch vector defined in Equation (2).

The strain $\Delta \varepsilon_{i j}$ of the particulate medium is related to the relative movement between the particles $\Delta \delta_{i}{ }^{n m}$ under the assumption of uniform strain field in the media. Thus

$$
\Delta \delta_{\mathrm{i}}^{\mathrm{nm}}=l_{\mathrm{j}}^{\mathrm{nm}} \Delta \varepsilon_{\mathrm{ij}}
$$


In Equation (9), the strain tensor $\Delta \varepsilon_{\mathrm{ij}}$ is not necessarily symmetrical. The non-symmetrical part of the strain tensor represents the relative spinning of the particles (Chang, 1989). For the case where the strain is symmetrical (Walton, 1987; Batchelor \& O'Brien, 1977), the effect of relative spinning of particles is neglected.

Combining Equations (3) and (8), the stress $\Delta \sigma_{i j}$ can be written in terms of the relative movement between particles $\Delta \delta_{i}{ }^{n m}$. Using Equation (9), the stiffness tensor $C_{\mathrm{ijk},}$ in Equation (2) which relates the stress and strain can be derived. For a random packing with a large number of contacts, the stress-strain tensor $C_{\mathbf{i j k}}$ in Equation (2) can be reasonably expressed in form of an integral by introducing a continuous distribution of branch vectors. Considering a packing made of equally sized particles, the branch vector distribution is reduced to the contact normal distribution defined by the density function $\xi(\boldsymbol{n})$. Thus Equation (2) can be restated as (Chang \& Misra, 1989a)

$$
C_{\mathrm{ijk1}}=\frac{2 N \rho^{2}}{V} \int_{\Omega} n_{\mathrm{j}}{ }^{\mathrm{nm}} n_{1}{ }^{\mathrm{nm}} K_{\mathrm{ik}}{ }^{\mathrm{nm}} \xi(\boldsymbol{n}) \mathrm{d} \Omega
$$

where $\rho$ is the radius of a particle, $N$ is the total number of contacts (each contact counted twice) in volume $V$ and $\mathrm{d} \Omega=\sin \gamma \mathrm{d} \gamma \mathrm{d} \beta$ with the integration limit as $0 \leqslant \gamma \leqslant \pi$ and $0 \leqslant \beta \leqslant 2 \pi, \gamma$ and $\beta$ being defined in Fig. 1. Thus the parameters $(N / V), \rho$ and $\xi(n)$ represent the geometrical characteristics of the particulate medium while $K_{\mathrm{ik}}$ represents the particle and contact properties.

\section{CONTACT FORCE-DISPLACEMENT LAW \\ Normal contact stiffness $K_{n}$}

Relative movement of two topographically smooth non-conforming deformable, elastic, bodies in contact as a result of normal force was studied by Hertz (Johnson, 1985). However, in natural sands the particles are inelastic, nonlinear, not perfectly rounded and have rough contact surface. To account for these factors, the normal stiffness for the contact may be written as a power law

$$
K_{\mathrm{n}}=C_{1} f_{\mathrm{n}}^{\alpha}
$$

where $C_{1}$ is a function of the material and surface properties and the particle size, $f_{\mathrm{n}}$ is the normal force at the contact and $\alpha$ is an exponent. In the Hertz theory, $\alpha=\frac{1}{3}$ and $C_{1}=\left(3 \rho E^{2}\right)^{1 / 3}$, where $E=G /(1-v)$ and $\rho$ is the particle radius. Parameters $C_{1}$ and $\alpha$ may take different values to account for the contact conditions which usually differ from the ideal Hertzian hypothesis.

The Hertz theory was extended by Johnson (1976) to include the effect of adhesion at the contact. The presence of adhesive forces at the contact results in an increase of contact area and alteration of contact pressure distribution. Considering adhesive forces at the contact, Johnson (1976) derived the relationship between the relative compression of two spheres and the contact force. Based on his results the normal stiffness for such contacts can be obtained as

$$
K_{\mathrm{n}}=\frac{96 E^{2} a^{4}\left(\chi_{\mathrm{c}}+f_{\mathrm{n}}\right)}{40 E \chi_{\mathrm{c}} a^{3}+32 E f_{\mathrm{n}} a^{3}-3 \rho f_{\mathrm{n}}^{2}}
$$

where $a$ is the radius of the contact surface given by

$$
a=\left(\frac{3 \rho}{8 E}\left[f_{\mathrm{n}}+2 \chi_{\mathrm{c}}+2 \sqrt{ }\left(f_{\mathrm{n}} \chi_{\mathrm{c}}+\chi_{\mathrm{c}}^{2}\right)\right]\right)^{1 / 3}
$$

and $\chi_{\mathrm{c}}$ is the contact adhesion force. When $f_{\mathrm{n}}=$ $-\chi_{c}$ (i.e. the contact tensile force equals the contact adhesion force) the contact separates (i.e. $K_{\mathrm{n}}=0$ ). For $\chi_{\mathrm{c}}=0$ (i.e. contact with no adhesion) Equations (12) and (13) reduce to that given by Hertz theory for non-adhesive contacts.

\section{Tangential contact stiffness $K_{\mathrm{r}}$}

For a contact of two topographically smooth spheres, the tangential stiffness under oscillating contact force was studied by Mindlin and Deresiewicz (1953). The tangential force at the contact causes deformation due to the development of slip over a part of the contact surface. When the tangential force exceeds the frictional strength at the contact; sliding takes place. Considering the partial slip, Mindlin and Deresiewicz derived the relationship for the tangential stiffness at the contact under various loading conditions. Based on their results, a general expression for the tangential stiffness of two dissimilar non-linear inelastic rough particles can be written as a function of the contact force and the particle properties as follows

$$
K_{\mathrm{r}}=C_{2} K_{\mathrm{n}}\left(1-\frac{f_{\mathrm{r}}}{f_{\mathrm{n}} \tan \phi_{\mu}}\right)^{\eta}
$$

where $C_{2}$ and $\eta$ are material constants, $\phi_{\mu}$ is particle-to-particle friction angle and $f_{\mathrm{r}}$ is the resultant tangential force at the contact. For the case of topographically smooth spheres the value of

$$
C_{2}=\frac{2(1-v)}{2-v} \text { and } \eta=\frac{1}{3}
$$

For contacts with adhesion, the slip at the contact is resisted by the adhesion as well as by friction. Sliding occurs when the tangential contact force exceeds the total strength, including adhesion and friction. The tangential stiffness for 
adhesive contacts can be obtained by modifying Equation (14) as follows

$$
K_{\mathrm{r}}=C_{2} K_{\mathrm{n}}\left(1-\frac{f_{\mathrm{r}}}{\left(\chi_{\mathrm{c}}+f_{\mathrm{n}}\right) \tan \phi_{\mu}}\right)^{\eta}
$$

An oscillating tangential force results in load reversals at the contact. Three types of tangential loading condition can be identified at the contact: (a) virgin loading - the first-time loading; (b) unloading - the loading reversal in the direction opposite to that of the virgin loading; and (c) reloading - the loading reversal that leads to loading in the direction of the virgin loading. Under a tangential force, the shear tractions are distributed on the contact area in a parabolic shape such that the tractions are large near the edges and decrease towards the centre. However, the slip resistance follows the normal traction distribution, that is it is low near the edges and increases towards the centre to form an inverted parabola. Thus, slippage occurs near the edge of the contact and therefore, near the edges, the shear traction distribution follows the slip resistance distribution. A section through the shear traction distribution on a contact surface during virgin loading is shown in Fig. 2 by the envelope $P Q R S$. In portions $P Q$ and $R S$ of the envelope, the shear tractions have reached the slip resistance at the contact. The annular area between circles $A$ and $B$ is slipping, whereas the area within the circle B is sticking. Immediately on load reversal (unloading), the shear traction over the contact area becomes less than the slip resistance such that the complete contact area sticks. Continued load reversal results in an increase of the shear tractions in the opposite direction which leads to counter-slip in the opposite direction to the initial slip. Thus a new slip annulus develops, starting from the edge of the contact area. For example, in the case of unloading, the shear force distribution envelope is given by PUVWXS with portions PU and XS having reached the slip resistance. The new slip area is the annulus between circles $A$ and $\mathbf{B}^{\prime}$ with the slip occurring in a direction opposite to the original slip.

The tangential stiffness is also affected by the oscillation of the normal force, as the oscillation of normal force leads to the oscillation of resistance to slip. Descriptions of the non-adhesive contacts under various combinations of oscillating tangential and normal contact forces have been developed by Mindlin and Deresiewicz (1953). A concise form of the equations by Mindlin and Deresiewicz has been given by Thornton and Randall (1987). These equations are modified here to account for adhesive contacts and the expressions for the tangential stiffness applicable to the various cases are given by

$$
K_{\mathrm{r}}=C_{2} K_{\mathrm{n}}\left(\frac{1}{\theta} \mp \frac{\Delta f_{\mathrm{n}} \tan \phi_{\mu}}{\Delta f_{\mathrm{r}}}\left[\frac{1}{\theta}-1\right]\right)^{-1}
$$

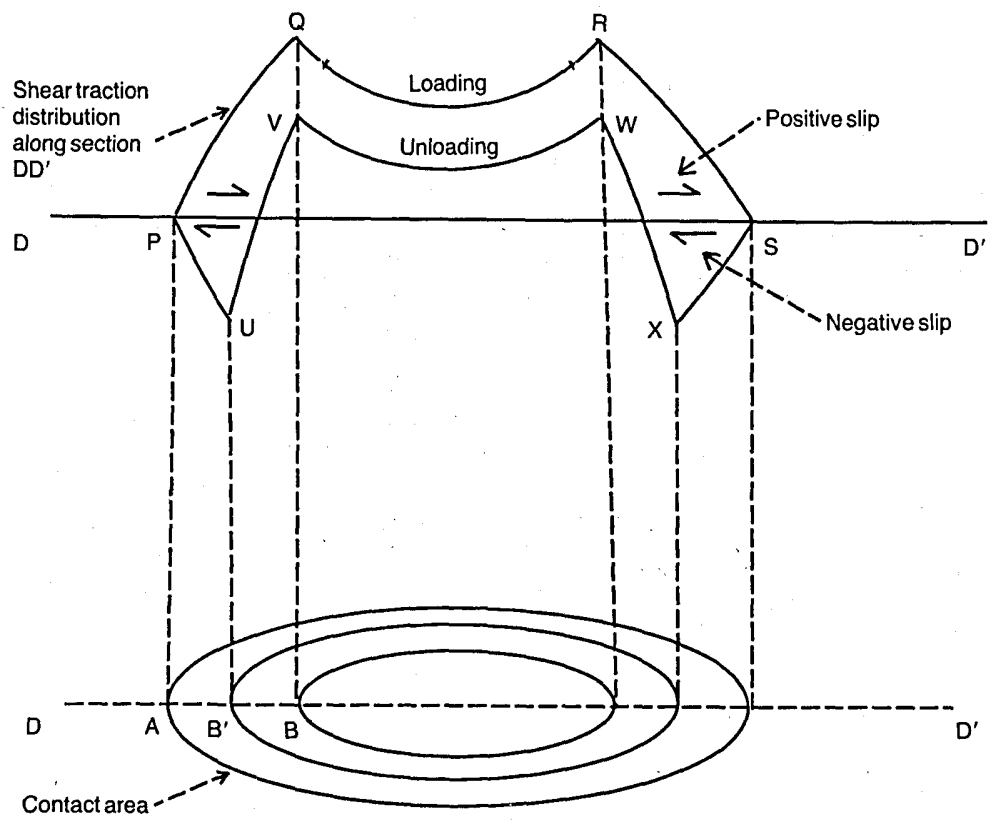

Fig. 2. Slip annulus at contact 
where the positive sign is invoked during unloading only, $C_{2}$ and $K_{\mathrm{n}}$ are given by Equations (15) and (12) respectively and

$$
\begin{aligned}
& \theta^{3}=1-\left(\frac{f_{\mathrm{r}}}{\left(\chi_{\mathrm{c}}+f_{\mathrm{n}}\right) \tan \phi_{\mu}}+\frac{\Delta f_{\mathrm{n}}}{f_{\mathrm{n}}}\right) \\
& \text { (virgin loading) } \\
& \theta^{3}=1-\left(\frac{f_{\mathrm{r}}^{*}-f_{\mathrm{r}}}{2\left(\chi_{\mathrm{c}}+f_{\mathrm{n}}\right) \tan \phi_{\mu}}+\frac{\Delta f_{\mathrm{n}}}{f_{\mathrm{n}}}\right) \\
& \theta^{3}=1-\left(\frac{f_{\mathrm{r}}^{* *}-f_{\mathrm{r}}}{2\left(\chi_{\mathrm{c}}+f_{\mathrm{n}}\right) \tan \phi_{\mu}}+\frac{\Delta f_{\mathrm{n}}}{f_{\mathrm{n}}}\right)
\end{aligned}
$$$$
\text { (reloading) }
$$

where $f_{\mathrm{r}}^{*}$ and $f_{\mathrm{r}}^{* *}$ are the loading and unloading reversal points respectively as shown in Fig. 3. For the case when $\Delta f_{\mathrm{n}} \tan \phi_{\mu}>\Delta f_{\mathrm{r}}$, the value of $\theta$ is taken to be 1 until $\Sigma \Delta f_{n} \tan \phi_{u} \leqslant \Sigma \Delta f_{\mathrm{r}}$.

In addition to the above equations, discussion of another situation is necessary in order to account for the stress history. Subsequent to a virigin loading, whenever the loading direction changes, the slip restarts from the edge of the contact area and propagates inwards. The inner radius of the slip annulus is given by

$$
\begin{aligned}
& B=A\left(1-\frac{f_{\mathrm{r}}^{*}-f_{\mathrm{r}}}{2\left(\chi_{\mathrm{c}}+f_{\mathrm{n}}\right) \tan \phi_{\mu}}\right)^{1 / 3} \\
& B=A\left(1-\frac{f_{\mathrm{r}}-f_{\mathrm{r}}^{* *}}{2\left(\chi_{\mathrm{c}}+f_{\mathrm{n}}\right) \tan \phi_{\mu}}\right)^{1 / 3}
\end{aligned}
$$

(reloading)

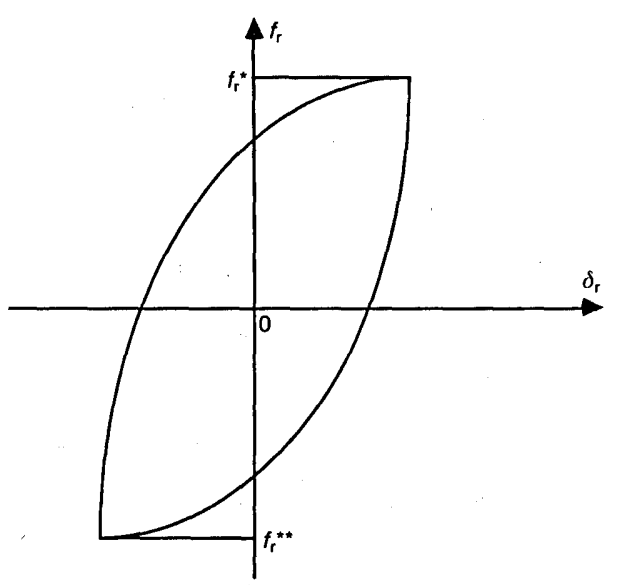

Fig. 3. Curve for contact displacement relationship under oscillating tangential force
Whether it is in the unloading or reloading state, when the value of $B$ becomes less than the previous minimum $B$, the slip is fully reversed. Therefore the subsequent loading is the same as the virgin loading condition and the stiffness should be given by Equation (18).

\section{MODEL PARAMETERS}

It is assumed that the cemented sand is modelled as randomly packed spheres with adhesion at contacts. The parameters needed for the model are: $(a)$ number of contacts per unit volume $(N / V) ;(b)$ the branch vector density function $\xi(\gamma, \beta) ;(c)$ the inter-particle friction angle $\phi_{\mu} ;(d)$ the contact adhesion $\chi_{\mathrm{c}} ;(e)$ the particle shear modulus $G ;(f)$ the particle Poisson's ratio $v ;(g)$ the particle radius $\rho$. Parameters $(a)$ and $(b)$ define the packing structure, parameters $(c)$ and $(d)$ define the particle contact properties, and parameters $(e)-(g)$ are the particle properties.

\section{Packing structure}

The packing structure is characterized by the number of contacts per unit volume $N / V$ and the spatial distribution of the contact normals $\xi(\gamma, \beta)$.

The number of contacts (doubly counted) per unit volume $N / V$ for a packing of spheres can be obtained from the void ratio, the co-ordination number and the particle size as

$$
(N / V)=\left(3 \bar{n} / 4 \pi \rho^{3}(1+e)\right)
$$

The void ratio $e$ is defined as the ratio of the volume of voids to the volume of solids, the coordination number $\vec{n}$ is defined as the average number of contact points per particle, and $\rho$ is the particle radius. The relationship between the co-ordination number $\bar{n}$ and the void ratio $e$ has been experimentally studied for assemblies of lead shot (Smith, Foote \& Busang, 1929), glass balls (Oda, 1977), rockfill materials (Marsal, 1973), and gravels (Yanagisawa, 1983). Based on the experimental data, the relationship between the void ratio and the co-ordination number is approximated, for the range of $0.38 \leqslant e \leqslant 0.87$ by

$$
e=1 \cdot 66-0 \cdot 125 \bar{n}
$$

Fig. 4 shows Equation (24) plotted with the experimental data.

The spatial distribution of contact normals is in general a function of angles $\gamma$ and $\beta$ shown in Fig. 1. The form of the density function $\xi(\gamma, \beta)$. can be best expressed by spherical harmonic expansion (Chang and Misra, 1989a). For simplicity, the sands considered are assumed to have an isotropic distribution of contact normals. For a 


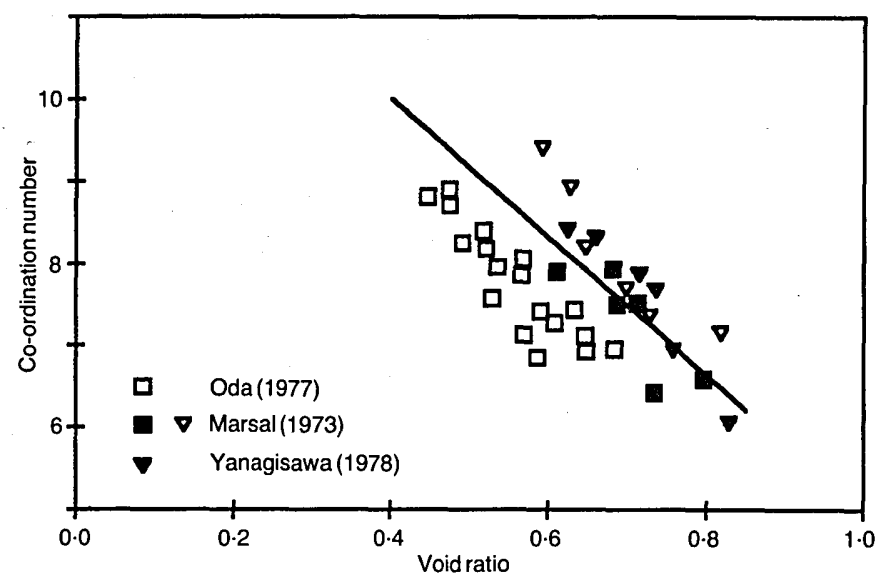

Fig. 4. Relationship between co-ordination number and void ratio

statistically isotropic packing, the density function $\xi(\gamma, \beta)$ is independent of $\gamma$ and $\beta$, given by

$$
\xi(\gamma, \beta)=1 / 4 \pi
$$

\section{Particle properties}

The particle properties taken into account in the computations are the particle shear modulus $G$, Poisson's ratio $v$, and the particle radius $\rho$. The particle shear modulus and the Poisson's ratio depend on the mineral composition of the particles. The sands considered in this Paper, namely crushed medium sand, Ottawa sand, mortar sand, Monterey No. 0 sand and Sengenyama sand, are all quartz sands. For these sands, the typical values of shear modulus $G$ and Poisson's ratio $v$ are given in Table 1 .

The particle radius $\rho$ is taken to be the mean particle radius of the soil. The soil is assumed to be a packing of equal sized spheres. This is justifiable because all the sands considered here are relatively uniformly graded. The values of particle radius $\rho$ are given for three sands in Table 1 .

\section{Contact properties}

Contact properties consist of the contact adhesion $\chi_{\mathrm{c}}$ and the inter-particle friction angle $\phi_{\mu}$. The inter-particle friction angle $\phi_{\mu}$ is a constant for a given mineral type. Contact friction $\phi_{\mu}$ is selected for a smooth surface mineral-to-mineral friction angle. Values of $\phi_{\mu}$ used in the predictions are given in Table 1.

The contact adhesion $\chi_{c}$ is related to the degree of cementation, the type of cementing agent, the composition of the sand and the curing period. In this study, only fully cured cemented sands are considered and the effect of curing period is not taken into consideration. The contact adhesion $\chi_{c}$ is determined empirically using the experimentally measured initial shear modulus $G_{\max }$ under an isotropic confining stress. The values of $\chi_{c}$ for various $D_{\mathrm{c}}$ are calculated based on the theoretical relationship of the initial shear modulus $G_{\max }$ obtained from Equation (10) for a statistically isotropic packing under an isotropic confining stress. From Equation (10), using Equations (12), (16), (23) and (25), the initial shear modulus $G_{\max }$ is derived to be

$$
G_{\max }=\frac{4 \cdot 8 E^{2} a^{4} \bar{n}\left(2+3 C_{2}\right)\left(\chi_{\mathrm{c}}+f_{\mathrm{n}}\right)}{\pi \rho(1+e)\left(40 E \chi_{\mathrm{c}} a^{3}+32 E f_{\mathrm{n}} a^{3}-3 \rho f_{\mathrm{n}}{ }^{2}\right)}
$$

Where $a$ is the contact radius given by Equation (13), $E=G /(1-v), C_{2}$ is defined in Equation (15), $\rho$ is the particle radius, and $\bar{n}$ and $e$ are the coordination number and void ratio, respectively, related through Equation (24). For a statistically isotropic packing under an isotropic stress state, the shear forces vanish $\left(f_{\mathrm{r}}=0\right)$ at all contacts and the normal forces $f_{\mathrm{n}}$ are of the same magnitude at all contacts given by (Chang et al., 1989)

$$
f_{\mathrm{n}}=\left(4 \pi \rho^{2}(1+e) / \bar{n}\right) \sigma_{0}
$$

where $\sigma_{0}$ is the isotropic confining stress.

The values of $\chi_{c}$ for various $D_{c}$, back calculated from Equation (26) using the experimentally measured initial shear modulus for four types of cemented sands, are shown by symbols in Fig. 5 . The measured initial shear modulus $G_{\max }$ for all the sands are at a confining stress of $\sigma_{0}=14.2 \mathrm{psi}$ $(98 \mathrm{kPa})$. The four cement-sand mixtures are as follows (Chang, 1986; Acar \& El-Tahir, 1986): lime cement and medium crushed sand, sodium silicate cement and Ottawa sand, fly ash-cement and mortar sand, and Portland cement and Mon- 
Table 1. Model parameters for three types of sand-cement mixtures

\begin{tabular}{|c|c|c|c|}
\hline \multirow[t]{2}{*}{ Parameters } & \multicolumn{3}{|c|}{ Sand-cement mixture } \\
\hline & $\begin{array}{l}\text { Crushed medium with lime cement } \\
\text { (Chang \& Woods, 1987) }\end{array}$ & $\begin{array}{l}\text { Monterey No. } 0 \text { with Portland cement } \\
\text { (Acar \& El-Tahir, 1986) }\end{array}$ & $\begin{array}{l}\text { Sengenyama with no cement } \\
\text { (Iwasaki \& Tatsuoka, 1977) }\end{array}$ \\
\hline $\begin{array}{l}\text { Particle shear modulus } G: \mathrm{kPa} \\
\text { Poisson's ratio } v \\
\text { Particle diameter } 2 \rho: \mathrm{mm} \\
\text { Contact friction } \phi_{\mu} \\
\text { Contact adhesion } \chi_{\mathrm{c}} \text { (from Equation (26)) } \\
\lambda_{1} \\
\lambda_{2} \\
\lambda_{3} \\
\text { Void ratio } e \\
\text { Co-ordination No. } \bar{n} \\
\text { Distribution function } \xi(\gamma, \beta)\end{array}$ & $\begin{array}{c}3.5 \times 10^{6} \\
0.13 \\
1.52 \\
10^{\circ} \\
\\
6.80 \\
60 \\
-2 \\
0.67 \\
\text { From Equation (24) } \\
\text { From Equation (25) }\end{array}$ & 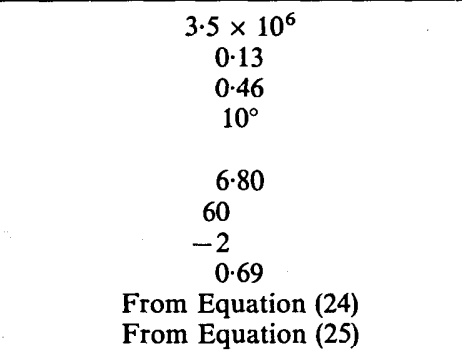 & 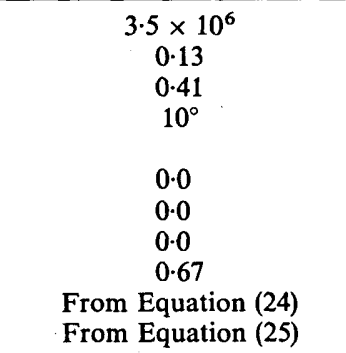 \\
\hline
\end{tabular}



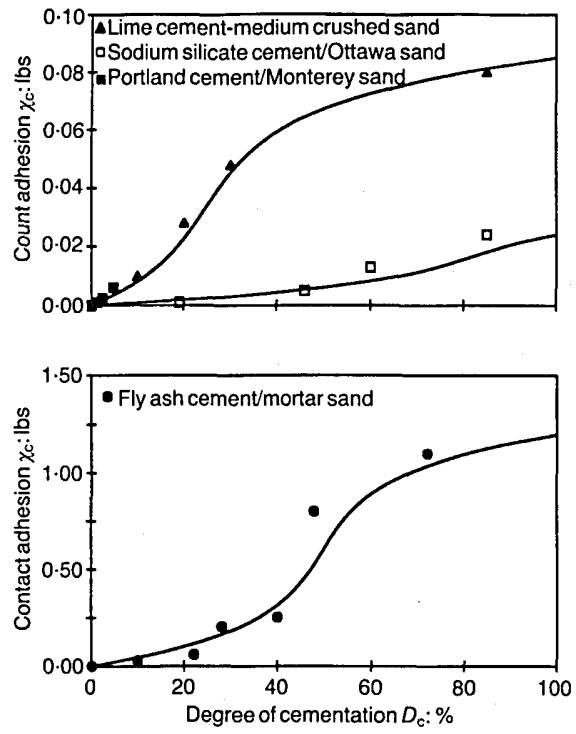

Fig. 5. Relationship between contact adhesion and degree of cementation

terey No. 0 sand. The parameters, particle shear modulus $G$, Poisson's ratio $v$, particle radius $\rho$, void ratio $e$ and co-ordination number $\bar{n}$, used in this computation are given in Table 1 for two of the cemented sands.

From the back calculated values of contact adhesion $\chi_{c}$ it can be observed that a typical relationship of contact adhesion $\chi_{\mathrm{c}}$ against degree of cementation $D_{c}$ consists of three distinctive parts. In the first stage, of low degree of cementation (up to about $8 \%$ ), the contact adhesion is relatively low as the cement coats the particles to form weak bonds at the particle contacts. In the second stage (from about 8 to $35 \%$ ), that is with further addition of cement, the contact adhesion increases rapidly with degree of cementation because stronger and bigger bonds are formed at the particle contacts. At a higher degree of cementation, which is the third stage, the added cement does not affect the bonds between the particles and merely fills up the void space. To represent such characteristics, the relationship between contact adhesion $\chi_{c}$ and degree of cementation $D_{c}$ can be expressed by an arcsine curve

$$
D_{c}=\lambda_{1}\left(\sinh \left(\lambda_{2} \chi_{c}+\lambda_{3}\right)-\sinh \lambda_{3}\right)
$$

The degree of cementation $D_{c}$ is the volume of cement per unit void volume of the sand. The fitted curves of $\chi_{c}$ against $D_{c}$ (Equation (28)) for these cemented sands are also shown in Fig. 5. The constants $\lambda_{1}, \lambda_{2}$ and $\lambda_{3}$ determined from the curve fitting are given in Table 1 .

\section{PROPERTIES OF CEMENTED SANDS}

The model is applied to study the properties of the cement-sand mixtures under low amplitude vibration, such as the initial tangent moduli, the secant moduli and the damping ratios. The model parameters for three types of cement-sand mixture studied: (a) crushed medium sand with lime cement (Chang \& Woods, 1987); (b) Monterey No. 0 sand with Portland cement (Acar \& El-Tahir, 1986) and (c) Sengenyama sand with no cement (Iwasaki \& Tatsouka, 1977), are given in Table 1. Using the values of parameters in Table 1 , the behaviour of initial tangent moduli, secant moduli, and damping ratio for the three types of sands are obtained from the mathematical model. These calculated behaviours from the model are compared with the available experimental measurements to examine the applicability of the model.

\section{Initial tangent moduli}

The initial tangent modulus $G_{\max }$, defined as the shear modulus under the initial stress condition is influenced by a number of factors such as the degree of cementation, the void ratio, the particle size and the initial confining stress (Equation (26)).

Effect of degree of cementation. Using the material parameters for the crushed medium sand and lime cement (Table 1), the variations of computed initial shear modulus with cement content $D_{\mathrm{c}}$ are shown in Fig. 6 for confining stresses of $7 \cdot 1 \mathrm{psi}(49 \mathrm{kPa})$ and $28.4 \mathrm{psi}(196 \mathrm{kPa})$. The experimental results for crushed medium sand and lime cement (Chang \& Woods, 1987) are shown by symbols in Fig. 6. The initial shear modulus $G_{\max }$ varies with the degree of cementation $D_{c}$ in two stages. At lower cement content $G_{\max }$ increases

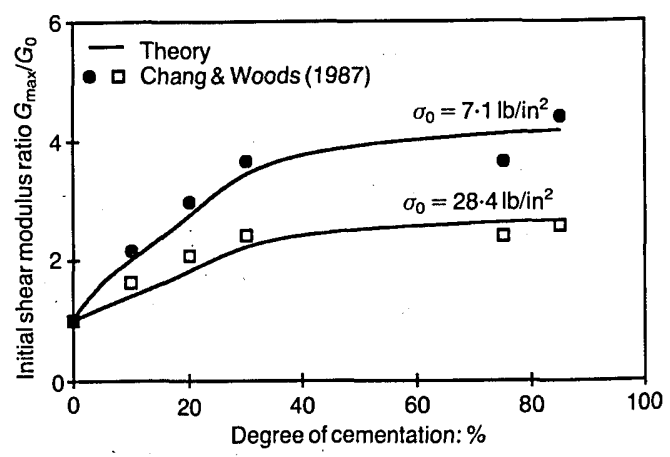

Fig. 6. Initial shear modulus ratio $G_{\max } / G_{0}$ against degree of cementation 
significantly with $D_{c}$ while at higher cement content the increase of $G_{\max }$ with $D_{\mathrm{c}}$ is small. This behaviour is due to the typical variation of contact adhesion $\chi_{\mathrm{c}}$ with degree of cementation $D_{c}$, shown in Fig. 5. Although the contact adhesion parameters were obtained from initial shear modulus for confining stress $\sigma_{0}=14.2 \mathrm{psi}$ $(98 \mathrm{kPa})$, the predicted results are in good agreement with results for $7.1 \mathrm{psi}(49 \mathrm{kPa})$ and $28.4 \mathrm{psi}$ $(196 \mathrm{kPa})$.

Effect of void ratio. The variation of $G_{\max }$ with the void ratio $e$ for Monterey No. 0 sand with Portland cement at initial confining stresses of 3 and $30 \mathrm{psi}(20.6$ and $206 \mathrm{kPa})$ and a degree of cementation of $5 \%$ is shown in Fig. 7. As expected the shear modulus is higher for dense sample.

Effect of particle size. Experimental studies on cemented sands (Chang, 1986) indicate that the shear modulus decreases with increasing particle size This is expected since for larger particles the average number of contacts per unit volume of the material is less. Therefore the number of adhesive bonds and the overall bonding force per unit volume is lower. The trend computed from the model is shown in Fig. 8 for the crushed medium sand with lime cement for degree of

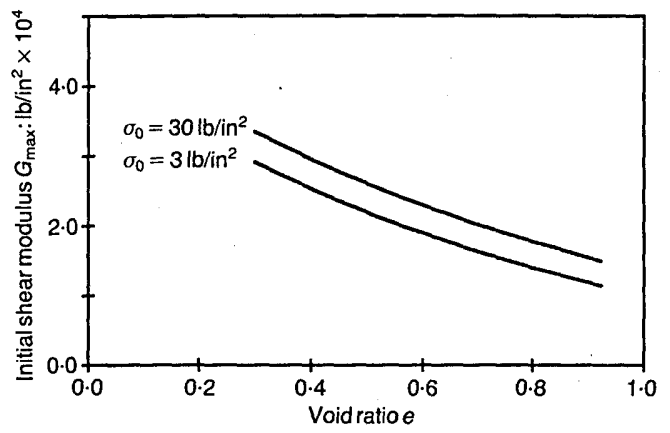

Fig. 7. Initial shear modulus against void ratio

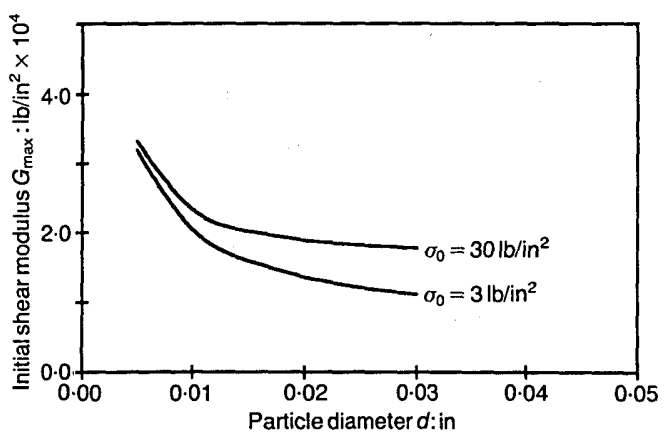

Fig. 8. Initial shear modulus against particle diameter cementation $6 \%$ and initial confining pressures of 3 and $30 \mathrm{psi}(20.6$ and $206 \mathrm{kPa})$. However, no direct measurements are available for comparison.

Effect of initial confining stress. The model prediction of the effect of initial confining stress $\sigma_{0}$ on the initial shear modulus $G_{\max }$ is shown in Fig. 9 for various degrees of cementation of crushed medium sand with lime cement. With the increase in the degree of cementation, the dependency of shear modulus on the confining stress decreases. This is because the contact adhesion is not a function of the confining stress and at a higher degree of cementation the contact adhesion has a dominating effect. Similar observations have been made by Chang \& Woods (1987) based on experimental results. However, they observe a higher slope of the $\log G_{\max }$ against $\log \sigma_{0}$. This discrepancy may be due to the idealization of real sand as an assembly of spherical, topographically smooth, linear elastic particles. To compare with the available test results the ratio $G_{\max } / G_{0}$ is plotted (Fig. 10) at different degrees of cementation against the initial confining stress for crushed medium sand with lime cement. $G_{\max }$ is the initial shear modulus of cemented sand and $G_{0}$ is the

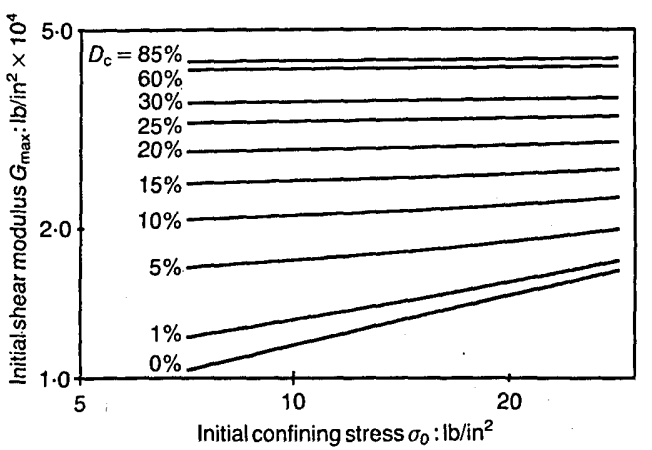

Fig. 9. Initial shear modulus against initial confining stress

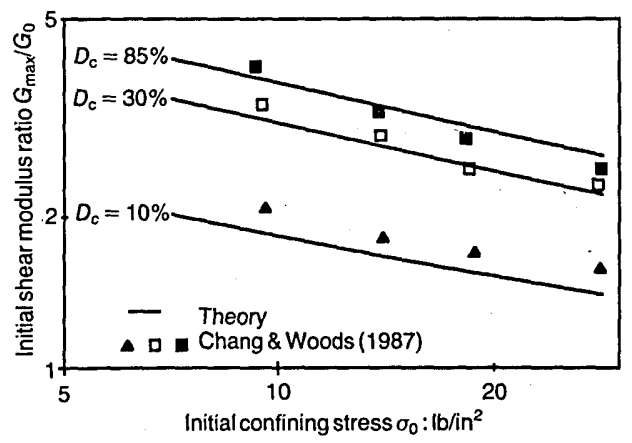

Fig. 10. Initial shear modulus ratio $G_{\max } / G_{0}$ against initial confining stress 


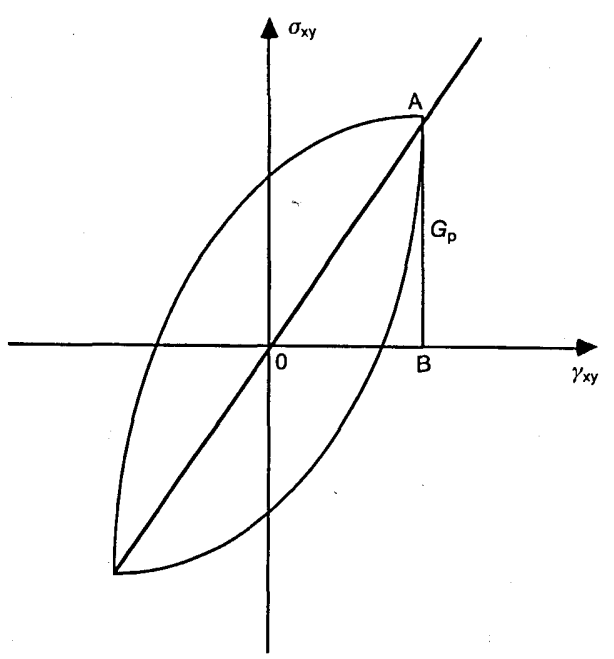

Fig. 11. Curve for shear stress against shear strain

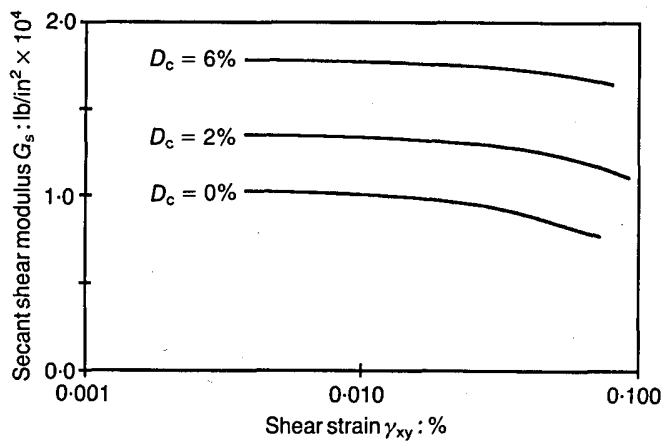

Fig. 12. Secant shear modulus against shear strain for various degrees of cementation initial shear modulus of uncemented sand. The prediction is shown by the continuous line and the measured data (Chang \& Woods, 1987) are shown by symbols.

\section{Secant moduli}

The secant shear modulus $G_{\mathrm{s}}$ is defined as the slope for a cyclic loop of shear stress, $\sigma_{x y}$ against shear strain $\gamma_{x y}$, as shown in Fig. 11. The effect of strain amplitude and initial confining stress on the secant moduli are discussed next.

Effect of strain amplitude. Fig. 12 shows the predicted variation of the secant shear modulus with the shear strain $\gamma_{x y}$, at degrees of cementation of 0,2 and $6 \%$ for Monterey No. 0 sand with Portland cement. As expected, the secant shear modulus $G_{\mathbf{s}}$ increases with degree of cementation. At very small strain levels, for a given degree of cementation, the secant shear modulus $G_{s}$ is almost constant up to threshold strain amplitude. For higher magnitude of strain amplitude, the secant shear modulus $G_{\mathrm{s}}$ decreases with increasing strain amplitude. This trend is reasonable, as indicated by the measured ratio of secant shear modulus and initial shear modulus $G_{\mathrm{s}} / G_{\max }$ (Acar \& El-Tahir, 1986). In Fig. 13, the predicted ratio $G_{\mathrm{s}} / G_{\max }$ is compared with the measured (Acar \& El-Tahir, 1986) ratio $G_{s} / G_{\max }$ for a degree of cementation of $2 \%$ under confining stresses of $7 \cdot 1$ and $14.2 \mathrm{psi}(48.98$ and $97.96 \mathrm{kPa})$. The predictions are shown by a continuous curve and the measured data by symbols. The measured values are almost same for the two confining pressures.

Effect of confining stress. To illustrate the effect of initial confining stress on the secant shear modulus $G_{s}$, the plots of $\log G_{\mathrm{s}}$ against $\log \sigma_{0}$ at

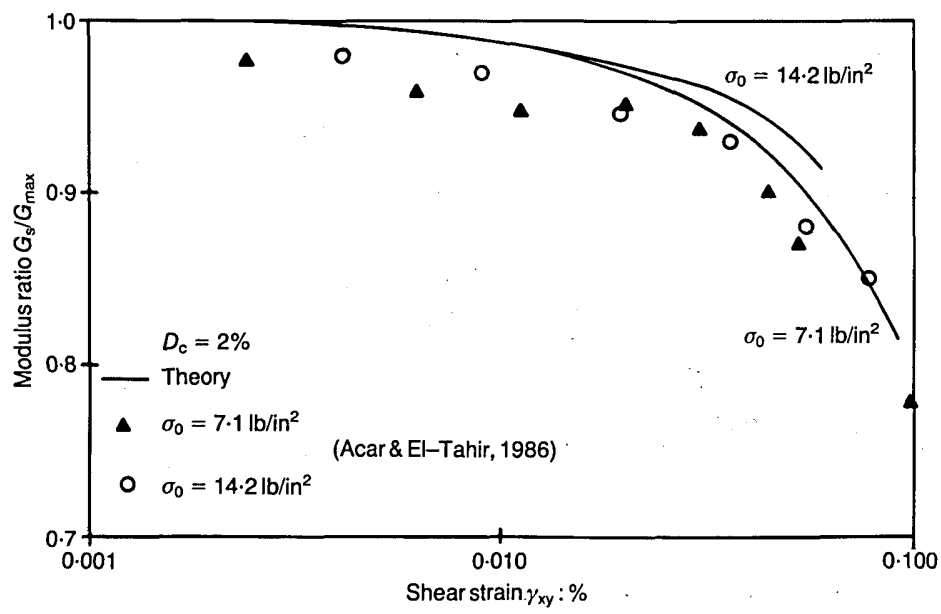

Fig. 13. Modulus ratio $G_{\mathrm{s}} / G_{\max }$ against shear stress 

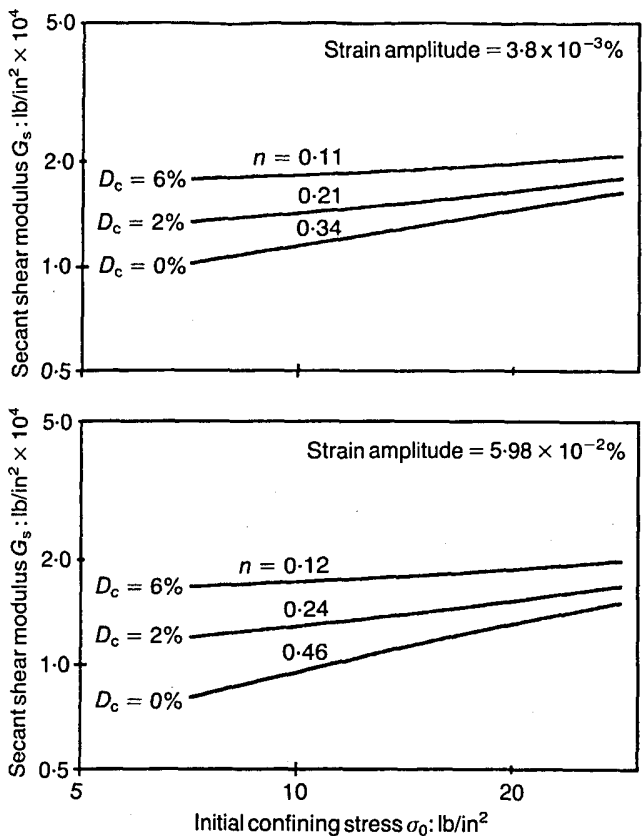

Fig. 14. Secant shear modulus against initial confining stress for different shear strain amplitudes with various degrees of cementation

shear strain amplitudes of $3.8 \times 10^{-3} \%$ and $5.98 \times 10^{-2} \%$ and at degrees of cementation of 0 , 2 and $6 \%$ for Monterey No. 0 sand with Portland cement are shown in Fig. 14. For a given strain amplitude, the slope of $\log G_{s}$ against $\log \sigma_{0}$ decreases with increasing degree of cementation. For a given degree of cementation, the slope increases with increasing strain amplitude. Increase of the slope with the increase in strain amplitude has also been observed for the case of clean sand (Iwasaki \& Tatsuoka, 1977). Fig. 15 gives the plot of $G_{\mathrm{s}}$ against $\sigma_{0}$ for two different

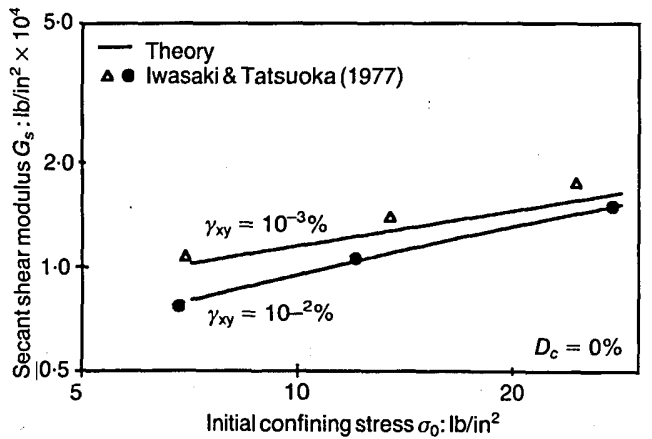

Fig. 15. Secant shear modulus against initial confining stress for different shear strain amplitudes with $0 \%$ cementation

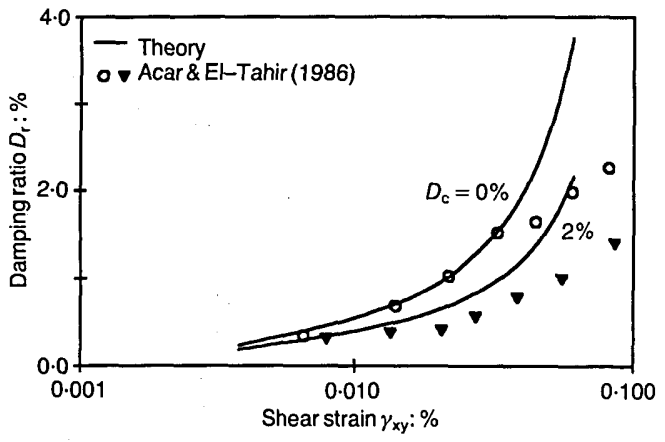

Fig. 16. Damping ratio against shear strain amplitude

strain amplitudes of the order $10^{-3}$ and $10^{-2} \%$, along with the experimental data for Sengenyama sands with no cement. The slope of the predicted results increases from 0.34 to 0.46 .

\section{Damping ratio}

The damping ratio is defined in Fig. 11 as

$$
D_{\mathrm{r}}=\frac{A_{\text {loop }}}{4 \pi A_{\mathrm{OAB}}}
$$

It is a measure of the energy dissipated during cyclic loading.

Effect of strain amplitude. The damping ratio is found to increase with increasing strain ampli-
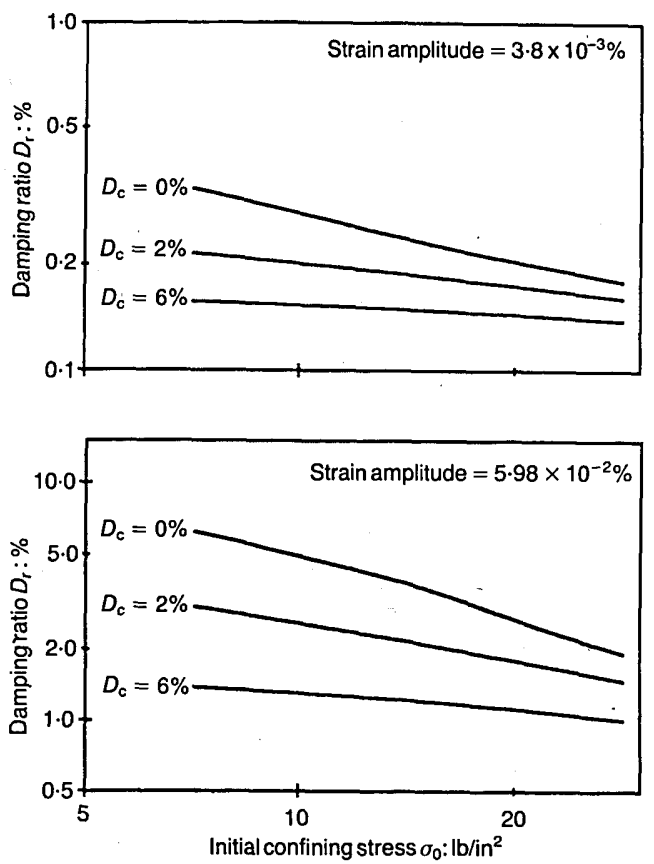

Fig. 17. Damping ratio against initial confining stress for different shear strain amplitudes with various degrees of cementation 
tude as illustrated in Fig. 16, which shows the variation of the damping ratio with strain amplitude at degrees of cementation of 0 and $2 \%$ under an initial confining stress of $14.2 \mathrm{psi}(97.96 \mathrm{kPa})$ for Monterey No. 0 sand with Portland cement. The predictions are shown by a continuous curve while the measured data (Acar \& El-Tahir, 1986) are shown by symbols. Fig. 16 also shows that the damping ratio is smaller for higher degree of cementation for a given strain amplitude. This is expected because an increase in the degree of cementation results in a stronger adhesion at the particle contacts, which leads to lower energy dissipation. The predicted damping ratio is found to increase more rapidly than the measured value. This indicates that the actual value of $\phi_{\mu}$ of the sand is, perhaps, higher than that assumed in the predictions.

Effect of confining stress. The damping ratio $D_{\mathrm{r}}$ is found to decrease with increase in the initial confining stress $\sigma_{0}$. Fig. 17 gives the plots of $\log$ $D_{\mathrm{r}}$ against $\log \sigma_{0}$ at strain amplitudes of $3.8 \times 10^{-3} \%$ and $5.98 \times 10^{-2} \%$ and at degrees of cementation of 0,2 and $6 \%$ for Monterey No. 0 sand and Portland cement. The slope of $\log D_{\mathrm{r}}$ against $\log \sigma_{0}$ is lower for higher degree of cementation. Also, for the same degree of cementation $D_{0}$, the slope is found to be higher for higher shear strain amplitude.

\section{CONCLUDING REMARKS}

The mechanical properties of cemented sands, such as initial tangent moduli, secant moduli and damping ratio, under small deformations are of interest in problems that involve low-amplitude oscillatory loading, such as seismic ground excitation, traffic loadings and vibration of machines on soil foundations. Here a microstructural continuum model for packing of spheres with frictional contacts is extended to consider the contact adhesion between two spheres. The model is then applied to study the dependency of these properties on degree of cementation, particle size, void ratio, initial confining stress and the strain amplitude. Although the microstructural model used in the analysis is rather idealized, based on isotropic packings of equal-sized spheres, the predicted behaviour for cemented sand agrees encouragingly well with both qualitative and quantitative observations from experiments. This approach, which treats sand as a collection of particles, is especially attractive because it is conceptually simple, realistic and explicitly accounts for factors such as: particle properties, the contact properties, the void ratio, the coordination number and the structural anisotropy. The model presented in this Paper is intended for small deformation $\left(<10^{-2} \%\right)$ conditions. For large deformations, accompanied by loss of contacts, some of the assumptions made in the theory will not hold.

\section{REFERENCES}

Acar, Y. B. \& El-Tahir, E. A. (1986). Low strain dynamic properties of artificially cemented sand. $J$. Geotech. Engng, Am. Soc. Civ. Engrs 112, 1001-1005.

Batchelor, G. W. \& O'Brien, R. W. (1977). Thermal or electrical conduction through a granular material, Proc. R. Soc. LondonA. 355, 313-333.

Chang, C. S. (1987). Micromechanical modelling of constitutive relations for granular material. In Micromechanics of granular materials, Eds M. Satake \& J. T. Jenkins, pp. 271-278, Amsterdam: Elsevier.

Chang, C. S. (1989). Constitutive modelling of granular materials as generalized continua with non-linear kinematic fields. In Powders and grains, Eds J. Biarez \& R. Gourves, pp. 311-319, Rotterdam: Balkema.

Chang, C. S. \& Misra, A. (1989a). Stress-strain behavior of sands-a microstructural approach. Proc. 4th Int. Symp. on Non-Nuclear Ammunition with Structures, Panama City Beach, Florida. 354-359.

Chang, C. S. \& Misra, A. (1989b). Theoretical and experimental study of regular packings of granules. J. Engng. Mech., Am. Soc. Civ. Engrs 115(4), 704 720.

Chang, C. S., Sundaram, S. S. \& Misra, A. (1989). Initial moduli of particulated mass with frictional contacts. Int. J. Numer. Analyt. Methods Geomech. 13, No. 6., 629-644.

Chang, T. S. (1986). Dynamic behavior of cemented sand. PhD dissertation, University of Michigan, Ann Arbor, Michigan.

Chang, T. S. \& Woods, R. D. (1987). Effect of confining pressure on shear modulus of cemented sand. In Developments in geotechnical engineering: soilstructure interaction, Ed. A. S. Cakmak, Vol. 43, pp. 193-208, Amsterdam: Elsevier.

Christoffersen, J., Mehrabadi, M. M. \& Nemat-Nasser, S. 1981). A micromechanical description of granular material behavior. J. Appl. Mech., Am. Soc. Mech. Engrs 48, No. 2, 339-344.

Clough, G. W., Sitar, N., Bachus, R. \& Rad, N. S. (1981). Cemented sands under static loading. J. Geotech. Engng., Am. Soc. Civ. Engrs 107, 799-817.

Dupas, J. M. \& Pecker, A. (1979). Static and dynamic properties of sand-cement. J. Geotech. Engng., Am. Soc. Civ. Engrs 105, 419-435.

Iwasaki, T. \& Tatsuoka, F. (1977). Effects of grain size and grading on dynamic shear moduli of sands. Soils and Fdns 17, No. 3, 19-35.

Johnson, K. L. (1985). Adhesion at the contact of solids. In Theoretical and applied mechanics, Ed. W. T. Koiter, pp. 133-143, Amsterdam: North Holland Publishing Company.

Johnson, K. L. (1985). Contact mechanics, London: Cambridge University Press.

Marsal, R. J. (1973). Mechanical properties of rockfill. In Embankment dam engineering, pp. 109-145, New York: John Wiley. 
Mindlin, R. D. \& Deresiewicz, H. (1953). Elastic spheres in contact under varying oblique forces. J. Appl. Mech., Am. Soc. Mech. Engrs 20, No. 3, 327-344.

Oda, M. (1977). Co-ordination number and its relation to shear strength of granular material. Soils and Fdns 17, No. 2, 29-42.

Saxena, S. K. \& Lastrico, R. M. (1978). Static properties of lightly cemented sands. J. Geotech. Engng., Am. Soc. Civ. Engrs 104, 1449-1463.

Smith, W. O., Foote, P. D. \& Busang, P. F. (1929). Packing of homogeneous spheres. Phys. Rev. 34, 1271-1274.

Thornton, C. \& Randall, C. W. (1987). Applications of theoretical contact mechanics to solid particle system simulations. In Micromechanics of granular materials, Ed M. Satake \& J. T. Jenkins, pp. 245252, Amsterdam: Elsevier.

Walton, K. (1987). The effective elastic moduli of a random packing of spheres. J. Mech. Phys. Solids 35, 213-226.

Yanagisawa, E. (1983). Influence of void ratio and stress condition on the dynamic shear modulus of granular media. In Advances in the mechanics and the flow of granular materials, Ed. M. Shahinpoor, pp. 947960, Houston: Gulf Publishing. 